\title{
Nash Tracking Game with Preview by State Feedback for Linear Continuous-Time Markovian Jump Systems
}

\author{
Gou Nakura
}

E-mail: gg9925_fiesta@ybb.ne.jp

\begin{abstract}
In this paper, we study Nash tracking problems for linear continuous-time Markovian jump systems over finite time intervals. We introduce double coupled differential equatuons (CDREs) to give solutions of the tracking problems with multi-players. Corresponding to the double coupled properties of the CDREs, the forms of preview compensator dynamics are double coupled. In this paper, we focus on the derivation of the necessary conditions for the solvability of the tracking problems by variational approach.

Key Words: Tracking with preview; Feedback Nash equilibrium; Soft-constrained games; Markovian jump systems; Double coupled Riccati differential equations; Double coupled preview compensator dynamics
\end{abstract}

\section{Introduction}

Markovian jump systems ([3, 6, 7, 9, 15, 27, 28, 33]) have abrupt random mode changes in their dynamics. The mode changes follow Markov processes. Such systems may be found in the area of power systems, manufacturing systems, communications, aerospace systems, finantial enginnering and so on. Such systems are classfied into continuous-time $([3,9,15,28])$ and discretetime $([6,7,27])$ systems. The optimal and $\mathrm{H}_{\infty}$ control theory has presented for each of these systems repectively $([3,7,15])$.

It is well known that, for design of tracking control systems, preview information of reference signals is very useful for improving the performance of the closed-loop systems, and much work has been done for preview control systems $([5,10,11,21,22,27,28,32])$.

With regard to Nash game theory, much work has been done $([1,2,4,8,12,14,16,17,18,35])$. W. A. van den Broek et al. have considered any robustness on multi-players Nash game and presented the control strategies in both cases of soft-constrained and hard-constrained game settings ([35]). Moreover H. Mukaidani at al. have developed stochastic Nash games theory, numerical algorithms for solving some sets of cross-coupled algebraic Riccati equations (CAREs), and their applications to large-scale systems $([16,17])$. In [17], H. Mukaidani has presented soft-constrained Nash game strategies for weakly coupled large-scale sys- tems considering stochastic uncertainties. Nash game can be applied to not only engineering systems but also finantial systems, ecologic systems and so on ([13] and so on.) Multi-players setting on Nash games can be regarded as to be equivalent to multi-agents, and so Nash game theory with multi-players can give one of bases for multi-agents systems design theory. These characteriscs of Nash games show the significance on systems design theory from both points of view of theories and their applications ([12] and so on.).

In this paper, we study soft-constrained Nash tracking games with preview for linear continuous-time Markovian jump systems over finite time intervals. Both Nash game theory and design theory for Markovian jump systems have not been applied only to mechanical systems but also to finantial systems (Refer to $[7,8,13,15]$ and so on.), and so it is thought that it is significant to consider Nash games for Markovian jump systems. From the point of view of noncausal tracking, Nash game theory has not been yet fully investigated. We consider the Nash tracking game considering the Nash tracking game with multi players considering the effects of exogenous disturbances. We introduce some sets of cross-coupled differential Riccati equations (CDREs) to give Nash equilibrium strategies and some sets of preview or noncausal compensator dynamics. Especially, in this paper, we investigate some properties which preview or noncausal compensator dynamics have.

In this paper, we focus on the derivation of the necessary conditions for the solvability of the Nash tracking game problems with preview. We introduce Lagrange multipliers and calculate the first variation with regard to the focused input to derive the necessary conditions for the solvability of Nash tracking game formulated in this paper. As a result, we necessarily obtain the pair of coupled Riccati differential equations and preview compensator dynamics.

We need to obtain solutions of the sets of CDREs to design state feedback controllers and preview compensators. Corresponding to the differences of performance indices for general $S$-platers, we need to introduce $S$ different forms of preview compensator dynamics. Originally the Riccati differential equations which give the necessary and sufficient conditions for the solvability of some optimal control problems for the Markovian jump 
systems are coupled with each other. By the consideration of Nash multi players settings, the CDREs considered in this paper are double coupled (or coupled and cross-coupled). Corresponding to these coupled properties, the preview compensator dynamics introduced in this paper are double coupled.

Finally we give numerical examples and verify the effectiveness of the tracking game theory presented in this paper.

Notations: Throughout this paper the superscript "'" stands for the matrix transposition, $\|\cdot\|$ denotes the Euclidean vector norm and $\|v\|_{R}^{2}$ also denotes the weighted norm $v^{\prime} R v$. $O$ denotes the matrix with all zero components.

\section{Problem Formulation}

Let $(\Omega, \mathcal{F}, \mathcal{P})$ be a probability space, where $\Omega$ is the sample space, $\mathcal{F}$ is a $\sigma$-algebra of a subset of $\Omega$ called events and $\mathcal{P}$ is the probability measure on $\mathcal{F}$. On this space, we consider the following linear continuoustime system with reference signal and Markovian mode transitions:

$$
\begin{gathered}
\dot{x}(t)=A\left(m_{t}\right) x(t)+\sum_{k=1}^{S} B_{2, k}\left(m_{t}\right) u_{k}(t) \\
+B_{3}\left(m_{t}\right) r_{c}(t)+E\left(m_{t}\right) w(t), \\
x(0)=x_{0}, m_{0}=i_{0} \\
z_{c, k}(t)=C_{1, k}\left(m_{t}\right) x(t)+\sum_{k=1}^{S} D_{12, k}\left(m_{t}\right) u(t) \\
+D_{13, k}\left(m_{t}\right) r_{c}(t)
\end{gathered}
$$

where $x \in \mathbf{R}^{n}$ is the state, $w \in \mathbf{R}^{p}$ is the exogenous disturbance, $u_{k} \in \mathbf{R}^{m}, k=1, \cdots, S$, are the control inputs, $z_{c, k} \in \mathbf{R}^{k_{c}}, k=1, \cdots, S$, are the controlled outputs and $r_{c}(\cdot) \in \mathbf{R}^{r_{c}}$ is a known or measurable reference signal. $x_{0}$ is a given initial state and $i_{0}$ is a given initial mode.

$\left\{m_{t}\right\}$ is a homogeneous Markov process with right continuous trajectories and taking values on the finite set $\phi=\{1,2, \cdots, N\}$ with the following stationary transition probabilities:

$$
\mathcal{P}\left\{m_{t+\Delta}=j \mid m_{t}=i\right\}=\left\{\begin{array}{cc}
\pi_{i j} \Delta+o(\Delta) & i \neq j \\
1+\pi_{i i} \Delta+o(\Delta) & i=j
\end{array}\right.
$$

where $\pi_{i j} \geq 0$ is the transition rate from the state $i$ to $j, i \neq j$, and $\pi_{i i}=-\sum_{j=1, j \neq i}^{N} \pi_{i j}<0$. Corresponding to each mode $i$, we define $A_{i}:=A\left(m_{t}=i\right), B_{2, i, k}:=$ $B_{2, k}\left(m_{t}=i\right), B_{3, i}:=B_{3}\left(m_{t}=i\right), E_{i}:=E\left(m_{t}=i\right)$ $C_{1, i, k}:=C_{1, k}\left(m_{t}=i\right), D_{12, i, k}:=D_{12, k}\left(m_{t}=i\right)$ and $D_{13, i, k}:=D_{13, k}\left(m_{t}=i\right)$, respectively. We assume that these matrices are constant for each $i$. We also assume that they are of compatible dimensions.
For this system (1), we assume the following conditions:

$$
\begin{array}{ll}
\text { A1: } & D_{12, i, k}^{\prime} C_{1, i, k}=O \\
\text { A2: } & D_{12, i, k}^{\prime} D_{12, i, k}>O
\end{array}
$$

The soft-constraind Nash tracking game problems we address in this section for the system (1) are to design control laws $u_{k}(\cdot) \in L_{2}[0, T], k=1, \cdots, S$, over the finite horizon $[0, T]$, using the information available on the known part of the reference signal $r_{c}(t)$ and minimizing the sum of the energy of $z_{c, k}(t), k=1, \cdots, S$, for the given initial condition $x_{0}$ and the worst case of the disturbances $w(t) \in \tilde{L}_{2}\left([0, T]: \mathbf{R}^{k}\right)$, and the equiribria condition of the optimized other inputs $u_{c, l}, l \neq k$, $1 \leq l \leq S$. We denote by $\tilde{L}_{2}\left([0, T]: \mathbf{R}^{k}\right)$ the space of nonanticipative signals. Considering the average of the performance indices over the statistics of the unknown part of $r_{c}$, we define the following performance index:

$$
\begin{aligned}
& J_{T, k}\left(u_{1}, \cdots, u_{k}, \cdots, u_{S}, w\right) \\
:= & \mathbf{E}\left\{\int_{0}^{T} \mathbf{E}_{\bar{R}_{s}}\left\{\left\|z_{c, k}(s)\right\|^{2}-\|w(s)\|_{V_{k}}^{2}\right\} d s\right\}
\end{aligned}
$$

where $V_{k}>O$ is some weight matrix for the disturbance $w(t) . \quad \mathbf{E}$ is the expectation with respect to $m_{t}$. $\mathbf{E}_{\bar{R}_{s}}$ means expectation over $\bar{R}_{s+h}, h$ is the preview length of $r_{c}(t)$, and $\bar{R}_{s}$ denotes the future information on $r_{c}$ at time $s$, i.e., $\bar{R}_{s}:=\left\{r_{c}(l) ; s<l \leq T\right\}$.

We consider the following Nash tracking game problem for the system (1) and the performance index (2). The Soft-Constrained Nash Tracking Game Problem with Fixed-Preview by State Feedback: It is assumed that, at the current time $t, r_{c}(s)$ is known for $s \leq \min (T, t+h)$, where $h$ is the preview length. Find $\left\{u_{k}^{*}\right\},\left\{w^{*}\right\}$ satisfying the following (saddle point) condition:

$$
\begin{aligned}
& J_{T, k}\left(u_{1}^{*}, \cdots, u_{k}^{*}, \cdots, u_{S}^{*}, w\right) \\
& \leq J_{T, k}\left(u_{1}^{*}, \cdots, u_{k}^{*}, \cdots, u_{S}^{*}, w^{*}\right) \\
& \quad \leq J_{T, k}\left(u_{1}^{*}, \cdots, u_{k}, \cdots, u_{S}^{*}, w^{*}\right)
\end{aligned}
$$

where the control strategy $u_{k}^{*}(t), 0 \leq t \leq T$, is based on the information $R_{t+h}:=\left\{r_{c}(l) ; 0 \leq l \leq t+h\right\}$ with $0 \leq h \leq T$.

\section{Design of Tracking Controllers}

In this section, we present the Nash tracking game strategies for linear continuous-time Markovian jump systems by state feedback.

Now we consider the following double coupled (or coupled and cross-coupled) Riccati differential equations:

$$
\begin{aligned}
\dot{X}_{i, k}+ & A_{i}^{\prime} X_{i, k}+X_{i, k} A_{i}+C_{1, i, k}^{\prime} C_{1, i, k} \\
& +X_{i, k} E_{i} V_{k}^{-1} E_{i}^{\prime} X_{i, k}
\end{aligned}
$$




$$
\begin{gathered}
-X_{i, k} \sum_{l=1}^{S} B_{2, i, l} \tilde{R}_{i, l}^{-1} \tilde{S}_{i, k}+\sum_{j=1}^{N} \pi_{i j} X_{j}=O, \\
i=1, \cdots, N, k=1, \cdots, S
\end{gathered}
$$

where

$$
\tilde{R}_{i, k}=D_{12, i, k}^{\prime} D_{12, i, k}, \tilde{S}_{i, k}(t)=B_{2, i, k}^{\prime} X_{i, k}(t) .
$$

We obtain the following necessary conditions for the solvability of the Nash tracking game problem with $S$ players by state feedback for (1) and (2) and a saddle point strategy for it.

Theorem 3.1 Consider the system (1) and the performance index (2). Suppose the conditions $\mathbf{A 1}$ and A2. Then, if the Soft-Constrained Nash Game Tracking Problem with Fixed-Preview by State Feedback for (1) and (2) is solvable, there exist matrices $X_{i, k}(t), i=1, \cdots, N, k=1, \cdots, S$, satisfying the conditions $X_{i, k}(T)=O$ such that the double coupled Riccati differential equations (3) hold over $[0, T]$. Moreover a saddle point strategy for the tracking game problem (1) and (2) is given by

$$
\begin{aligned}
& w^{*}(t)=V_{k}^{-1} E_{i, k}^{\prime} X_{i, k}(t) x(t)+\mathbf{C}_{\theta, i, k} \theta_{i, k}(t) \\
& \begin{array}{r}
u_{k}^{*}(t)=-\tilde{R}_{i, k}^{-1} \tilde{S}_{i, k}(t) x(t)-\mathbf{C}_{u, i, k} r_{c}(t) \\
-\mathbf{C}_{\theta u, i, k} \theta_{c, i, k}(t) \\
\text { for } i=1, \cdots, N \text { and } k=1, \cdots, S
\end{array}
\end{aligned}
$$

where

$$
\begin{gathered}
\mathbf{C}_{\theta, i, k}=V_{k}^{-1} E_{i, k}^{\prime}, \quad \mathbf{C}_{\theta u, i, k}=\tilde{R}_{i, k}^{-1} B_{2, i, k}^{\prime}, \\
\mathbf{C}_{u, i, k}=\tilde{R}_{i, k}^{-1} D_{12, i, k}^{\prime} D_{13, i, k} .
\end{gathered}
$$

$\theta_{i, k}(t), i=1, \cdots, N, k=1, \cdots, S, t \in[0, T]$, satisfies

$$
\left\{\begin{array}{c}
\dot{\theta}_{i, k}(t)=-\bar{A}_{c, i, k}^{\prime}(t) \theta_{i, k}(t)+\bar{B}_{c, i, k}(t) r_{c}(t) \\
+\sum_{l=1, l \neq k}^{S} X_{i, k} B_{2, i, l} \tilde{R}_{i, l}^{-1} B_{2, i, l}^{\prime} \theta_{i, l}(t) \\
-\sum_{j=1}^{N} \pi_{i j} \theta_{j, k}(t), \\
\theta_{i, k}(T)=0
\end{array}\right.
$$

where

$$
\begin{gathered}
\bar{A}_{c, i, k}=A_{i}+E_{i} V_{k}^{-1} E_{i}^{\prime} X_{i, k}-B_{2, i, k} \tilde{R}_{i, k}^{-1} \tilde{S}_{i, k} \\
\bar{B}_{c, i}=-\left(X_{i, k} B_{3, k}+C_{1, i, k}^{\prime} D_{13, i, k}\right)+\tilde{S}_{i, k}^{\prime} \mathbf{C}_{u, i, k} \\
+\sum_{l=1, l \neq k}^{S} X_{i, k} B_{2, k, l} \mathbf{C}_{u, k, l}
\end{gathered}
$$

and $\theta_{c, i, k}(t)$ is the 'causal' part of $\theta_{i, k}(\cdot)$ at time $t$. This $\theta_{c, i, k}$ is the expected value of $\theta_{i, k}$ over $\bar{R}_{s}$ and given by

$$
\left\{\begin{array}{c}
\dot{\theta}_{c, i, k}(s)=-\bar{A}_{c, i, k}^{\prime}(s) \theta_{c, i, k}(s)+\bar{B}_{c, i, k}(s) r_{c}(s) \\
+\sum_{l=1, l \neq k}^{S} X_{i, k} B_{2, i, l} \tilde{R}_{i, l}^{-1} B_{2, i, l}^{\prime} \theta_{c, i, l}(t) \\
\quad-\sum_{j=1}^{N} \pi_{i j} \theta_{c, j, k}(s), \\
t \leq s \leq t_{f}, \\
\theta_{c, i, k}\left(t_{f}\right)=0
\end{array}\right.
$$

where

$$
\left\{\begin{array}{cc}
t_{f}=t+h & \text { if } t+h<T \\
t_{f}=T & \text { if } t+h \geq T .
\end{array}\right.
$$

(Proof)

Necessity: It will be explained in detail in the next section. (Q.E.D.)

Remark 3.1 Corresponding to the double coupled (or coupled and cross-coupled properties of the Riccati equations (3), the preview compensator dynamics (7) are cross-coupled (or should be called coupled and crosscoupled). In order to introduce the preview information to the control inputs, we need to drive all preview compensators simultaneously utilizing the all $N \times S$ solutions of the cross-coupled differential Riccati equations with the receding terminal conditions $\theta_{c, i, k}\left(t_{f}\right)=0$.

Remark 3.2 Because we introduce the preview information of the reference signal $r_{c}(\cdot)$ by solving the preview compensator dynamics (7), each player need to know the information of the matrices $C_{1, i, l}, D_{12, i, l}$ and $D_{13, i, l}, l \neq k$, of the controlled output in the system (1) for the other players $l \neq k$ at time $t$ until $t+h$. This point is different from the causal cases where we introduce only the information of reference signals until current time $t$.

\section{Derivation of Necessary Conditions with Multi Platers Cases}

We investigate the derivation of the necessary conditions with multi players cases and Markovian mode transitions.

We assume that the signal $\left\{r_{c}(t)\right\}$ is known a priori for the whole time interval $t \in[0, T]$. Notice that, in this case, the expectation $\mathbf{E}_{\bar{R}_{s}}$ is not necessary. In this case, we can regard the dynamics

$$
\begin{aligned}
\dot{x}(t)=A\left(m_{t}\right) x(t)+ & \sum_{k=1}^{S} B_{2, k}\left(m_{t}\right) u_{k}(t) \\
& +B_{3}\left(m_{t}\right) r_{c}(t)+E\left(m_{t}\right) w(t)
\end{aligned}
$$

as the constraint over the time interval $[0, T]$. Then we can define the following Lagrangian

$$
\begin{aligned}
& \bar{J}_{T, k}\left(u_{1}, \cdots, u_{k}, \cdots, u_{S}, w\right) \\
& :=-\int_{0}^{T}\|w(s)\|_{V_{k}}^{2} d s \\
& +\mathbf{E}\left\{\int_{0}^{T}\left\{\left\|z_{c, k}(s)\right\|^{2}\right\} d s\right. \\
& \quad+\left\|C_{1, k}\left(m_{T}\right) x(T)+D_{12, k}\left(m_{T}\right) r_{c}(T)\right\|_{Q_{T, k}}^{2} \\
& \quad+\int_{0}^{T} 2 \lambda_{\left(m_{s}\right), k}^{\prime}(s)\left\{A\left(m_{s}\right) x(s)+E\left(m_{s}\right) w(s)\right. \\
& \left.\left.\quad+\sum_{k=1}^{S} B_{2, k}\left(m_{s}\right) u_{k}(s)+B_{3}\left(m_{s}\right) r_{c}(s)-\dot{x}(s)\right\} d s\right\}
\end{aligned}
$$


where $Q_{T, k} \geq O$ and $\lambda_{\left(m_{s}\right), k}(\cdot), k=1, \cdots, S$, are Lagrange multipliers. Using the relationship

$$
\begin{aligned}
& \mathcal{L}_{u_{k}}\left\{\lambda_{i, k}^{\prime}(t) x(t)\right\} \\
= & \dot{\lambda}_{i, k}^{\prime}(t) x(t)+\lambda_{i, k}^{\prime}(t) \dot{x}(t)+\sum_{j=1}^{N} \pi_{i j} \lambda_{i, k}^{\prime}(t) x(t)
\end{aligned}
$$

and Dynkin's formula, we obtain the following partial integration formula.

$$
\begin{aligned}
& \mathbf{E}\left\{\int_{0}^{T} \lambda_{m(s), k}^{\prime}(s) \dot{x}(s) d s\right\}=\left[\lambda_{i, k}^{\prime}(s) x(s)\right]_{0}^{T} \\
& -\mathbf{E}\left\{\int_{0}^{T} \dot{\lambda}_{m(s), k}^{\prime}(s) x(s)+\sum_{j=1}^{N} \pi_{m(s) j} \lambda_{j, k}^{\prime}(s) x(s) d s\right\}
\end{aligned}
$$

We calculate the first variation of $\bar{J}_{T, k}$ with regard to $x, u_{k}, w$ and $\lambda_{m(t), k}$ and obtain

$$
\begin{gathered}
\delta \bar{J}_{T, k} \\
=-\int_{0}^{T} 2 \delta w^{\prime} V_{k} w d s \\
+\mathbf{E}\left\{\int _ { 0 } ^ { T } 2 \delta x ^ { \prime } ( s ) C _ { 1 , k } ^ { \prime } ( m _ { s } ) \left\{C_{1, k}\left(m_{s}\right) x(s)\right.\right. \\
\left.\quad+D_{12, k}\left(m_{s}\right) u_{k}(s)+D_{13, k}\left(m_{s}\right) r_{c}(s)\right\} \\
+2 \delta u_{k}^{\prime}(s) D_{12, k}^{\prime}\left(m_{s}\right)\left\{C_{1, k}\left(m_{s}\right) x(s)\right. \\
\left.\quad+\sum_{l=1}^{S} D_{12, l}\left(m_{s}\right) u_{l}(s)+D_{13, k}\left(m_{s}\right) r_{c}(s)\right\} d s \\
+\delta x^{\prime}(T)\left\{C_{1, k}^{\prime}\left(m_{T}\right) Q_{T, k} C_{1, k}\left(m_{T}\right) x(T)\right. \\
\left.\quad+2 C_{1, k}^{\prime}\left(m_{T}\right) Q_{T, k} D_{13, k}\left(m_{T}\right) r_{c}(T)\right\} \\
+\int_{0}^{T} 2 \delta \lambda_{m(s), k}^{\prime}(s)\left\{A\left(m_{s}\right) x(s)+\sum_{l=1}^{S} B_{2, l}\left(m_{s}\right) u_{l}(s)\right. \\
\left.\quad+B_{3}\left(m_{s}\right) r_{c}(s)+E\left(m_{s}\right) w(s)-\dot{x}(s)\right\} d s \\
+\int_{0}^{T} 2\left\{\delta x^{\prime}(s) A^{\prime}\left(m_{s}\right)+\delta u_{k}^{\prime}(s) B_{2, k}^{\prime}\left(m_{s}\right)\right. \\
\left.\quad+\delta w^{\prime}(s) E^{\prime}\left(m_{s}\right)\right\} \lambda_{m(s), k}(s) d s \\
+2 \lambda_{m(T), k}^{\prime}(T) \delta x(T)+2 \lambda_{m(0), k}^{\prime}(0) \delta x(0) \\
\left.+\int_{0}^{T} 2 \dot{\lambda}_{m(s), k}^{\prime} \delta x(s) d s+2 \sum_{j=1}^{N} \pi_{m(s) j} \lambda_{j, k}^{\prime}(s) \delta x(s) d s\right\} .
\end{gathered}
$$

Let $\delta \bar{J}_{T, k}=0$ and then we obtain the following conditions:

(i) the conditions of optimality

$$
\begin{aligned}
& w^{*}(s)=V_{i, k}^{-1} E_{i}^{\prime} \lambda_{i, k}^{*}(s) \\
& \begin{array}{l}
u_{k}^{*}(s)=-\tilde{R}_{i, k}^{-1}\left\{D_{12, i, k}^{\prime} C_{1, i, k} x^{*}(s)\right. \\
\left.\quad+D_{12, i, k}^{\prime} D_{13, i, k} r_{c}(s)+B_{2, i, k} \lambda_{i, k}^{*}(s)\right\}(9)
\end{array}
\end{aligned}
$$

(ii) the canonical equations

$$
\dot{x}^{*}(s)=A_{i} x^{*}(s)+\sum_{l=1}^{S} B_{2, i, l} u_{l}^{*}(s)
$$

$$
\begin{array}{r}
+B_{3, i} r_{c}(s)+E_{i} w^{*}(s), \\
\dot{\lambda}_{i, k}^{*}(s)=-A_{i}^{\prime} \lambda_{i, k}^{*}(s)-C_{1, i, k}^{\prime}\left\{C_{1, i, k} x(s)+D_{12, i, k} u_{k}^{*}(s)\right. \\
\left.+D_{13, i, k} r_{c}(s)\right\}-\sum_{j=1}^{N} \pi_{i j} \lambda_{j, k}(s)
\end{array}
$$

(iii) the boundary conditions

$$
\begin{aligned}
\lambda_{i, k}(T)=C_{1, i, k}^{\prime} Q_{T} & C_{1, i, k} x(T) \\
& +2 C_{1, i, k}^{\prime} Q_{T} D_{13, i, k} r_{c}(T)
\end{aligned}
$$

From the boundary conditions (12), we set

$$
\lambda_{m(t), k}(t)=\theta_{k}\left(t, m_{t}\right)+X_{k}\left(t, m_{t}\right) x(t) .
$$

Then we have the worst case disturbance

$$
w^{*}(t)=V_{k}^{-1} E^{\prime}\left(\theta_{i, k}(t)+X_{i, k}(t) x(t)\right)
$$

from (8) and the control strategy

$$
\begin{array}{r}
u_{k}^{*}(t)=-\tilde{R}_{i, k}^{-1}\left\{\left(D_{12, i, k}^{\prime} C_{1, i, k}+B_{2, i, k} X_{i, k}(t)\right) x^{*}(t)\right. \\
\left.+D_{12, i, k}^{\prime} D_{13, i, k} r_{c}(t)+B_{2, i, k} \theta_{i, k}(t)\right\}
\end{array}
$$

from (9). Next we calculate

$$
\begin{aligned}
& \mathcal{L}_{u_{k}}\left\{\lambda_{i, k}^{\prime}(s) x(s)\right\} \\
= & \dot{\lambda}_{i, k}^{\prime}(s) x(s)+\lambda_{i, k}^{\prime}(s) \dot{x}(s)+\sum_{j=1}^{N} \pi_{i j} \lambda_{j, k}^{\prime}(s) x(s) \\
= & -\left(\theta_{i, k}^{\prime}(s)+x^{\prime}(s) X_{i, k}(s)\right) A_{i} x(s) \\
& -\left\{C_{1, i, k} x(s)+D_{12, i, k} u_{k}^{*}(s)+D_{13, i, k} r_{c}(s)\right\}^{\prime} C_{1, i, k} x(s) \\
& -\sum_{j=1}^{N} \pi_{i j}\left\{\theta_{j, k}^{\prime}(s)+x^{\prime}(s) X_{j, k}(s)\right\} x(s) \\
& +\left(\theta_{i, k}^{\prime}(s)+x^{\prime}(s) X_{i, k}(s)\right)\left\{A_{i} x(s)+\sum_{l=1}^{S} B_{2, i, l} u_{l}^{*}(s)\right. \\
& \left.\quad+B_{3, i} r_{c}(s)+E_{i} w^{*}(s)\right\} \\
& +\sum_{j=1}^{N} \pi_{i j}\left\{\theta_{j, k}^{\prime}(s)+x^{\prime}(s) X_{j, k}(s)\right\} x(s)
\end{aligned}
$$

and

$$
\begin{aligned}
& \mathcal{L}_{u_{k}}\left\{\theta_{i, k}^{\prime}(s) x(s)+x^{\prime}(s) X_{i, k}(s) x(s)\right\} \\
= & \dot{\theta}_{i, k}^{\prime}(s) x(s)+\theta_{i, k}^{\prime}(s) \dot{x}(s)+\dot{x}^{\prime}(s) X_{i, k}(s) x(s) \\
+ & x^{\prime}(s) \dot{X}_{i, k}(s) x(s)+x^{\prime}(s) X_{i, k}(s) \dot{x}(s) \\
& +\sum_{j=1}^{N} \pi_{i j}\left\{\theta_{j, k}^{\prime}(s) x(s)+x^{\prime}(s) X_{j, k}(s) x(s)\right\} \\
= & \dot{\theta}_{i, k}^{\prime}(s) x(s)+\theta_{i, k}^{\prime}(s)\left\{A_{i} x(s)+E_{i} w^{*}(s)\right. \\
& \left.+\sum_{l=1}^{S} B_{2, i, l} u_{l}^{*}(s)+B_{3, i} r_{c}(s)\right\} \\
+2 x^{\prime}(s) X_{i, k}(s)\left\{A_{i} x(s)\right. & +E_{i} w^{*}(s) \\
& \left.+\sum_{l=1}^{S} B_{2, i, l} u_{l}^{*}(s)+B_{3, i} r_{c}(s)\right\}
\end{aligned}
$$




$$
\begin{aligned}
& +x^{\prime}(s) \dot{X}_{i, k}(s) x(s) \\
& +\sum_{j=1}^{N} \pi_{i j}\left\{\theta_{j, k}^{\prime}(s) x(s)+x^{\prime}(s) X_{j, k}(s) x(s)\right\} .
\end{aligned}
$$

where we have used (8)-(11). Since the right hand sides of the two equalities (14) and (15) are equal, by the arbitrariness of $x$, with regard to the second order terms of $x$, we obtain the following set of coupled differential Riccati equations, which gives the necessary condition for the solvability of the Nash tracking gamw problem:

$$
\begin{aligned}
\dot{X}_{i, k}+ & A_{i}^{\prime} X_{i, k}+X_{i, k} A_{i}+C_{1, i, k}^{\prime} C_{1, i, k} \\
& +X_{i, k} E_{i} V_{k}^{-1} E_{i}^{\prime} X_{i, k} \\
& -X_{i, k} \sum_{l=1}^{S} B_{2, i, l} \tilde{R}_{i, l}^{-1} \tilde{S}_{i, k}+\sum_{j=1}^{N} \pi_{i j} X_{j}=O \\
& X_{i, k}(T)=C_{1, i, k}^{\prime} Q_{T, k} C_{1, i, k}
\end{aligned}
$$

Also, with regard to the first order terms of $x$ we obtain

$$
\left\{\begin{array}{c}
\dot{\theta}_{i, k}(t)=-\bar{A}_{c, i, k}^{\prime}(t) \theta_{i, k}(t)+\bar{B}_{c, i, k}(t) r_{c}(t) \\
+\sum_{l=1, l \neq k}^{S} X_{i, k} B_{2, i, l} \tilde{R}_{i, l}^{-1} B_{2, i, l}^{\prime} \theta_{i, l}(t) \\
-\sum_{j=1}^{N} \pi_{i j} \theta_{j, k}(t) \\
\theta_{i, k}(T)=2 C_{1, i, k}^{\prime} Q_{T, k} D_{13, i, k} r_{c}(T)
\end{array}\right.
$$

which give the preview compensator dynamics introducing the known information of the reference signal $r_{c}(\cdot)$ with the terminal conditions.

Remark 4.1 Notice that, in the case of $Q_{T, k}=O$, the terminal conditions for (16) and (17) reduce to $X_{i, k}(T)=O$ and $\theta_{i, k}(T)=0$. Also notice that, in this case, the form of the above Riccati differential equations (16) with terminal conditions is equivalent to the form of the cross-coupled Riccati differential equations introduced for the Nash game problems without considering any reference signals nor preview information for the continuous-time Markovian jump systems [18].

Remark 4.2 As described in Remark 3.1, the preview compensator dynamics (7) in the fixed preview case has the same form as the noncausal compensator dynamics (6) in the noncausal case. We can obtain the preview compensator dynamics (7) with the receding terminal conditions $\theta_{c, i, k}\left(t_{f}\right)=0$ by restricting the final tiem $T$ in the Lagrangian $\bar{J}_{T, k}$ to $t+h$.

\section{$5 \quad$ Numerical Examples}

In this section, we study numerical examples to demonstrate the effectiveness of the design theory presented in this paper.

We consider the following two modes systems with two players and assume that the system parameters are as follows: (cf. $[5,27,28,32]$ and so on.)

$$
\begin{gathered}
\dot{x}(t)=A\left(m_{t}\right) x(t)+\sum_{k=1}^{2} B_{2, k}\left(m_{t}\right) u_{k}(t) \\
\quad+B_{3} r_{c}(t)+E w(t) \\
x(0)=x_{0}, m_{0}=i_{0}, i=1,2 \\
z_{c, k}(t)=C_{1, k}\left(m_{t}\right) x(t)+\sum_{k=1}^{2} D_{12, k}\left(m_{t}\right) u(t)+D_{13} r_{c}(t)
\end{gathered}
$$

- Mode 1:

$$
\begin{gathered}
A_{1}=\left[\begin{array}{cc}
0 & 1 \\
-1 & -0.4
\end{array}\right], \quad A_{2}=\left[\begin{array}{cc}
0.5 & 1 \\
0.8 & -0.2
\end{array}\right] \\
B_{2,1,1}=\left[\begin{array}{l}
0 \\
1
\end{array}\right], B_{2,1,2}=\left[\begin{array}{c}
0 \\
0.5
\end{array}\right], E=\left[\begin{array}{c}
1 \\
-1
\end{array}\right], \\
B_{2,2,1}=\left[\begin{array}{c}
0 \\
1
\end{array}\right], B_{2,2,2}=\left[\begin{array}{c}
0 \\
0.5
\end{array}\right], B_{3}=\left[\begin{array}{c}
1 \\
0
\end{array}\right], \\
C_{1, i, 1}=\left[\begin{array}{cc}
-0.5 & 0.1 \\
0 & 0
\end{array}\right], i=1,2, \\
C_{1, i, 2}=\left[\begin{array}{c}
-0.5 \\
0 \\
0
\end{array}\right], i=1,2, \\
D_{12, i, 1}=\left[\begin{array}{c}
0 \\
0.1
\end{array}\right], \begin{array}{c}
D_{12, i, 1}=\left[\begin{array}{c}
0 \\
0.2
\end{array}\right], i=1,2, \\
D_{13}=\left[\begin{array}{c}
-1.0 \\
0
\end{array}\right]
\end{array} \\
{\left[\begin{array}{c}
0.2 \\
0
\end{array}\right]}
\end{gathered}
$$$$
\text { Mode 2: }
$$

where it is assumed that $r_{c}(\cdot)$ is not always a priori known over the whole time interval $[0, T]$ but has any distribution at the unknown part. Let

$$
\Pi=\left[\begin{array}{cc}
-0.2 & 0.2 \\
0.3 & -0.3
\end{array}\right]
$$

be a transition matrix of $\left\{m_{t}\right\}$. Let the weight matrices $V_{1}=I$ and $V_{2}=I$. We set $x_{0}=\operatorname{col}(0,0)$ and $i_{0}=1$.

Then we introduce the following objective function considering the input energies:

$$
\begin{aligned}
& J_{T, k}\left(x_{0}, u_{1}, u_{2}, w\right) \\
= & \mathbf{E}\left\{\int _ { 0 } ^ { T } \mathbf { E } _ { \overline { R } _ { s } } \left\{\left\|C_{1, k}\left(m_{s}\right) x(s)+D_{13} r_{c}(s)\right\|^{2}\right.\right. \\
& \left.\left.+\sum_{k=1}^{2}\left\|D_{12, k} u_{k}(s)\right\|^{2}\right\} d s\right\}
\end{aligned}
$$

By the term $B_{3} r_{c}(t)$, the tracking performance can be expected to be improved as $[5,27,28,32]$ and so on. 


\section{Concluding Remarks}

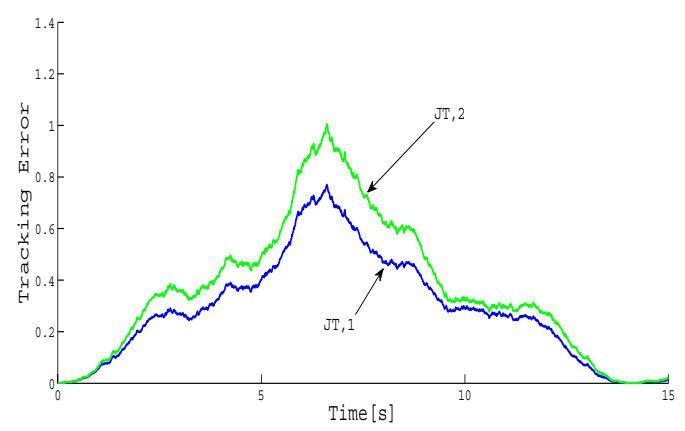

Fig. 1: The value of tracking error for $h=0.5$

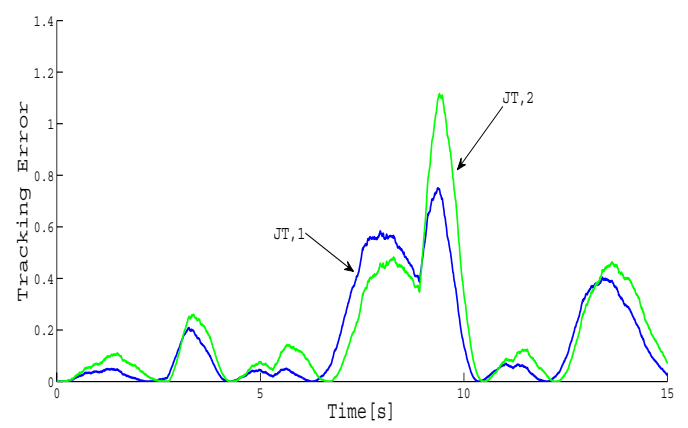

Fig. 2: The value of tracking error for $h=0.75$

The paths of $m_{t}$ are generated rondomly, and the performances are compared under the same circumstance, that is, the same set of the paths so that the performances can be easily compared.

We condider the whole system (18) with mode transition rate $\Pi$ over the time interval $t \in[0,15]$. For this system, we apply the results of the soft-constrained Nash tracking game theory for $r_{c}(t)=\sin (\pi t / 15)$ with two lengths of preview $h=0.5$ and $h=0.75$. We verify the effectiveness of the preview compensation by state feedback, and compare the tracking performances for $k=1,2$. We define We define

$$
\begin{aligned}
& T E_{k}\left(r_{c}(t), h\right) \\
:= & \mathbf{E}\left\{\frac{1}{T} \int_{0}^{T} \sqrt{\left\|C_{1, k}\left(m_{s}\right) x(k)+D_{13} r_{c}(s)\right\|^{2}} d s\right\}
\end{aligned}
$$

for each $k$ and $h$.

Fig. 1 shows that the values of $J_{T, 1}$ and $J_{T, 2}$ for $h=0.5$. Fig. 2 shows the values of $J_{T, 1}$ and $J_{T, 2}$ for $h=0.75$. We also obtain the values $T E_{1}(0.5)=$ $0.4810, T E_{2}(0.5)=0.5390, T E_{1}(0.75)=0.3310$ and $T E_{2}(0.75)=0.3690$. These simulation results show that we can obtain better performances for $h=0.75$ than $h=0.5$ in both indices $J_{T, 1}$ and $J_{T, 2}$ numerically.
In this paper, we have studied the soft-constrained Nash tracking game with preview for the linear continuous-time Markovian jump systems, which are a class of stochastic switching systems by state feedback. Both Nash game and tracking control with preview are of high practice. We have presented the Nash tracking strategies with preview compensators.

In order to give Nash tracking strategies with preview, we have introduced cross-coupled differential Riccati equations (CDREs). Utilizing the set of solutions of the CDREs, we have designed the state feedback controllers with preview compensators, which give the Nash tracking problems over the finite time intervals.

In this paper, we have mainly considered the derivation of the necessary conditions for the Nash game problems with preview. We have calculated the first variation of the performance indices under the constraint of the system dynamics to derive the necessary conditions for the solvability of the Nash tracking game.

In order to obtain Nash tracking game strategies with preview, we have introduced double coupled (or coupled and cross-coupled) differential Riccati equations (CDREs). Utilizing the set of solutions of the CDREs, we have designed the state feedback controllers with preview compensators, which give the Nash tracking problems over the finite time intervals. Corresponding to the double coupled properties of the Riccati differential equations, we have obtained the double coupled (or coupled and cross coupled) preview compensator dynamics.

Finally we have studied the numerical examples to compare the tracking control performances. For discrete-time Markovian jump systems, some Nash tracking theories will be reported elsewhere.

\section{References}

[1] T. Basar and G. Olsre: Dynamic Noncooperative Game Theory, Warrendale, PA: SIAM, 1995.

[2] T. Basar: Paradigms for Robustness in Controller and Filter Design, Proceedings of the IFAC Conference on Modeling and Control of Economics Systems, Klagenfurt, Austria, pp.11-17, 2001.

[3] E. K. Boukas: Stochasting Switching Systems: Analysis and Design, Birkhauser, Boston, 2006.

[4] R. Buckdahn, P. Cardaliaguet and C. Rainer: Nash Equlibrium Payoffs for Nonzero-Sum Stochastic Dufferential Games, SIAM J. Control, Optim., Vol. 43, No. 2, pp. 624-642, 2004.

[5] A. Cohen and U. Shaked: Linear Discrete-Time $\mathrm{H}_{\infty}$-Optimal Tracking with Preview, IEEE Trans. Automat. Contr., Vol. 42, No. 2, pp. 270-276, 1997. 
[6] O. L. V. Costa: Linear Minimum Mean Square Error Estimation for Discrete-Time Markovian Jump Linear Systems, IEEE Trans. Automat. Contr., Vol. 39, No. 8, pp.1685-1689, 1994.

[7] O. L. V. Costa, M. D. Fragoso and R. P. Marques: Discrete-Time Markov Jump Linear Systems, Springer, London, 2005.

[8] J. C. Engwerda: LQ Dynamic Optimization and Differential Games, New York: Wiley, 2005.

[9] M. D. Fragoso, O. L. V. Costa, J. Baczynski and N. Rocha: Optimal Linear Mean Square Filter for Continuous-Time Jump Linear Systems, IEEE Trans. Automat. Contr., Vol. 50, No. 9, pp.13641369, 2005.

[10] E. Gershon, D. J. N. Limebeer, U. Shaked and I. Yaesh: Stochastic $\mathrm{H}_{\infty}$ Tracking with Preview for State-Multiplicative Systems, IEEE Trans. Automat. Contr., Vol. 49, No. 11, pp. 2061-2068, 2004 .

[11] E. Gershon, U. Shaked and I. Yaesh, $\mathrm{H}_{\infty}$ tracking of linear continuous-time systems with stochastic uncertainties and preview, Int. J. Robust and Nonlinear Control, Vol. 14, No. 7, pp. 607-626, 2004.

[12] D. Gu: A Differential Game Approach to Formulation Control, IEEE Trans. Control Systems Technology, Vol. 16, No. 1, 2008.

[13] L. P. Hansen, T. J. Sargent and T. D. Tallarini, Jr.: Robust Permanent Income and Pricing, Review of Economic Studies, Vol. 66, pp. 873-907, 1999.

[14] D. J. N. Limebeer, B. D. O. Anderson and B. Hendel: A Nasg Game Approach to Mixed $\mathrm{H} 2 / \mathrm{H}_{\infty}$ Control, IEEE Trans. Automat. Contr., Vol. 39, pp. 69-82, 1994.

[15] M. Mariton: Jump Linear Systems in Automatic Control, Marcer Dekker, New York, 1990.

[16] H. Mukaidani: A Numerical Analisys of the Nash Strategy for Weakly Coupled Large-Scale Systems, IEEE Trans. Automat. Contr., Vol. 51, pp. 13711377, 2006.

[17] H. Mukaidani: Soft-Constrained Stochastic Nash Games for Weakly Coupled Large-Scale Systems, Automatica, Vol. 45, No. 5, pp. 1272-1279, 2009.

[18] H. Mukaidani, M. Unno, H. Xu and V. Dragan: Nash Strategies of Markov Jump Stochastic Systems Applied to Weakly-Coupled LargeScale Systems, Proceedings of the 18th IFAC World Congress, Milano (Italy), 2011.

[19] G. Nakura: $\mathrm{H}_{\infty}$ Tracking with Preview for Linear Continuous-Time Markovian Jump Systems,
Proceedings of the SICE 8th Annual Conference on Control Systems, Kyoto, Japan, 073-2-1 (CDROM), 2008.

[20] G. Nakura: Noncausal Optimal Tracking for Linear Switched Systems, Proceedings of The 11th International Workshop of Hybrid Systems : Computation and Control (HSCC2008), St. Louis, MO, USA (2008, 4, 22-24), Springer LNCS Vol.4981, pp. 372-385, 2008.

[21] G. Nakura: $\mathrm{H}_{\infty}$ Tracking with Preview for Linear Systems with Impulsive Effects -State Feedback and Full Information Cases-, Proceedings of the 17th IFAC World Congress, Seoul, Korea, 2008, TuA08.4 (CD-ROM).

[22] G. Nakura: $\mathrm{H}_{\infty}$ Tracking with Preview by Output Feedback for Linear Systems with Impulsive Effects, Proceedings of the 17th IFAC World Congress, Seoul, Korea, TuA08.5 (CD-ROM), 2008.

[23] G. Nakura: $H_{\infty}$ Tracking with Preview for Linear Discrete-Time Markovian Jump Systems, Proceedings of the 37th Symposium on Control Theory, Kirishima, Japan, pp.171-176, 2008.

[24] G. Nakura: Stochastic Optimal Tracking with Preview for Linear Discrete-Time Markovian Jump Systems by Output Feedback with Known Modes, Proceedings of the ICROS-SICE International Joint Conference 2009 (ICCAS-SICE2009), 2A062 (CD-ROM), Fukuoka, Japan, 2009.

[25] G. Nakura: On Noncausal $\mathrm{H}_{\infty}$ Tracking Control for Linear Continuous-Time Markovian Jump Systems, Proceedings of the 41st ISCIE International Symposium on Stochastic Systems Theory and Its Applications (SSS09), Kobe, Japan, pp. 172-177, 2009.

[26] G. Nakura: On Noncausal $\mathrm{H}_{\infty}$ Tracking Control for Linear Discrete-Time Markovian Jump Systems, Proceedings of the 19th IEEE International Conference on Control Applications (CCA 2010), Yokohama, Japan, FrA10.4, pp. 1981-1986 (CDROM), 2010.

[27] G. Nakura: Stochastic Optimal Tracking with Preview by State Feedback for Linear Discrete-Time Markovian Jump Systems, International Journal of Innovative Computing, Information and Control (IJICIC), Vol. 6, No. 1, pp. 15-27, 2010.

[28] G. Nakura: Stochastic Optimal Tracking with Preview by State Feedback for Linear ContinuousTime Markovian Jump Systems, SICE Journal of Control, Measurement and System Integration (SICE JCMSI), Vol. 3, No. 3, pp. 164-171, 2010. 
[29] G. Nakura: An Approach to Hybrid Smoothing for Linear Continuous-Time Systems with NonGaussian Noises, Proceedings of the 43rd ISCIE International Symposium on Stochastic Systems Theory and Its Applications (SSS11), Shiga, Japan, pp. 63-72, FB2-2, (CD-ROM), 2011.

[30] G. Nakura: On Noncausal $\mathrm{H}_{\infty}$ Tracking Control for Linear Impulsive Systems with Stochastic Uncertainties, Trans. of The Institution of Systems, Control and Information Engineers, Vol. 26, No. 12, pp. 456-465, 2013.

[31] G. Nakura: Stochastic Optimal Tracking with Preview for Linear Continuous-Time Markovian Jump Systems by Output Feedback, Trans. of The Institution of Systems, Control and Information Engineers, Vol. 29, No. 11, pp. 497-505, 2016.

[32] U. Shaked and C. E. de Souza: Continuous-Time Tracking Problems in an $\mathrm{H}_{\infty}$ Setting: A Game Theory Approach, IEEE Trans. Automat. Contr., Vol. 40, No. 5, pp.841-852, 1995.

[33] A Survey on Markovian Jump Systems: Modeling and Design, Int. J. Control, Automation, Systems, Vol. 13, No. 1, pp. 1-16, 2005.

[34] D. D. Sworder and R. O. Rogers: An LQG Solution to a Control Problem with Solar Thermal Receiver, IEEE Trans. Automat. Contr., Vol. 28, pp.971-978, 1983.

[35] W. A. Van Den Broek, J. C. Engwerda and J. M. Schumacher: Robust Equilibria in Indefinite Linear-Quadratic Differential Games, J. Optimization Theory and Applications, Vol. 119, No. 3, pp. 565-595, 2003. 